\title{
Light-modulated scanning tunneling spectroscopy for nanoscale imaging of surface photovoltage
}

\author{
Osamu Takeuchi, Shoji Yoshida, and Hidemi Shigekawa ${ }^{a)}$ \\ Institute of Applied Physics, 21st century COE, University of Tsukuba 1-1-1 Tennodai, Tsukuba, \\ Ibaraki 305-8573, Japan
}

(Received 16 May 2003; accepted 12 March 2004; published online 20 April 2004)

\begin{abstract}
Light-modulated scanning tunneling spectroscopy (LM-STS) is proposed as a useful method for investigating spatially resolved surface photovoltage (SR-SPV). LM-STS provides the dependences of SR-SPV on bias voltage under constant tip-sample distance simultaneously with the entire dark/illuminated $I-V$ curves. With this method, it is shown that SPV of a metallic $\mathrm{Si}(111)$ surface can be bias-dependent and $\mathrm{SPV}$ at zero bias voltage for $\mathrm{Si}(001)$ can be tip-sample-distance-dependent under conditions of small tip-sample distance and high illumination intensity. The importance of the experimental condition for interpreting experimentally obtained SR-SPV was suggested. (C) 2004 American Institute of Physics. [DOI: 10.1063/1.1737063]
\end{abstract}

Since the invention of scanning tunneling microscopy (STM), many studies have been devoted to the utilization of STM for measuring surface photovoltage (SPV). ${ }^{1-10}$ In general, SPV is defined as the change in surface potential of a semiconductor material caused by superband gap illumination and it provides important electric properties of the material, such as the polarity and magnitude of surface band bending, the recombination rate of photocarriers, and surface conductivity. ${ }^{11}$ Thus, acquiring spatially resolved SPV (SRSPV) simultaneously with an atomic topograph by STM will lead to a greater understanding of the electric functions of nanodevices on the basis of their atomic structures.

Since the pioneering work by Hamers and Markert, ${ }^{2}$ several experimental methods in two categories have been developed, namely, for measuring intrinsic (zero bias) $\mathrm{SPV}^{2-5}$ and SPV under finite bias voltages, ${ }^{6-9}$ using STM. It is assumed that SPV alters the effective sample bias voltage at the tunnel junction. Hence, the lateral shift of $I-V$ characteristics of the junction caused by the illumination is measured as SR-SPV. In the measurement of the intrinsic SPV, the disappearance of the differential tunnel conductance $d I / d V$ at low bias voltages enhanced the small error in tunnel current detection, resulting in a large noise/artifact of the obtained intrinsic SPV. Thus, SR-SPV measurement is now often performed under finite bias voltages. In these cases, however, the local band structure beneath the STM probe can be affected by the strong electric field and high current density required for the STM observation, in addition to the effect of the contact potential difference between the probe and the sample. Hence, the result of previous studies are apparently conflicting to each other. For better understanding of the obtained SR-SPV, the measurement conditions must be carefully taken into consideration.

First, Cahill et al. reported that SPV measurement is sensitive to the tunnel current setting but not to the bias voltage; they interpreted this result using the concept of the charging effect. ${ }^{6}$ According to it, the tunnel current setting determines the amount of space charge under the STM probe and governs the deviation of SPV from the intrinsic value. However,

${ }^{a)}$ Electronic mail: http://dora.ims.tsukuba.ac.jp/
McEllistrem et al. reported that SPV is strongly dependent on bias voltage when the surface has a lower density of states at the Fermi level. ${ }^{7}$ It was suggested that, since the low metallic surface state does not screen the electric field of the STM probe, the penetration of the electric field into the subsurface region affects surface potential under a dark condition. On the other hand, Sommerhalter et al. insisted that both tunnel current and bias voltage affect surface potential when the surface has a negligible surface state density. ${ }^{10}$ In particular, they suggested that the tip-sample distance does not greatly affect the surface potential under a dark condition, but affects that under an illuminated condition.

In consideration of the importance of the SR-SPV study for the development of future electronic devices, suitable experimental conditions for SR-SPV measurement and a procedure for the analysis of the obtained results must be established. In order to clarify the disagreements among the previous results, exhaustive studies of the SPV dependence on experimental conditions will be required. In this letter, we propose a simple experimental method, light-modulated scanning tunneling spectroscopy (LM-STS), as a powerful method for such studies. LM-STS has two advantages. First, it gives the SPV dependence on experimental conditions complementary to what was obtained in previous methods. It measures the SPV dependence on bias voltage under the condition of constant tip-sample distance, in contrast to previous methods in which the condition of constant tunnel current is adopted. Second, it provides not only SR-SPV but also the complete illuminated and dark I-V curves simultaneously, providing a deeper insight into the sample properties. We apply the method to a metallic $\mathrm{Si}(111)-7 \times 7$ surface and a semiconductive $\operatorname{Si}(001)-2 \times 1$ surface, and revealed properties of SR-SPV. SR-SPV for the metallic Si(111)- $7 \times 7$ surface was strongly dependent on bias voltage, particularly in the low bias voltage regime. The SPV dependence on the tip-sample distance at zero bias voltage obtained for $\operatorname{Si}(001)-2 \times 1$ revealed a fundamental difficulty of measuring the intrinsic SPV by STM potentiometry.

LM-STS was performed as follows. Mechanically chopped illumination was provided from $45^{\circ}$ off-normal to the surface and focused onto a spot of $\sim 0.05 \mathrm{~mm}$ diameter at 
(a)

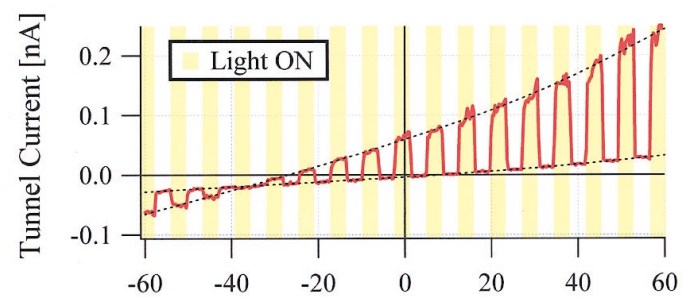

(b)

(c)

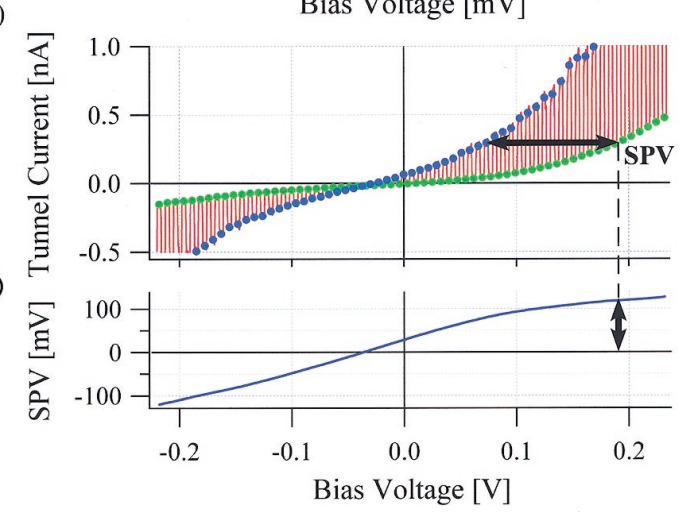

FIG. 1. (Color) Typical result of LM-STS measurement on $n$-type $\mathrm{Si}(111)$ $(30 \Omega \mathrm{cm})$. (a) Magnified plot of raw STS data under chopped illumination (red) and its upper/lower envelopes (dotted black). (b) Entire spectrum. (c) Extracted SPV spectrum.

the tunnel junction. The problematic thermal expansion/ shrinkage of the STM tip (electrochemically etched tungsten wire, $\phi 0.25 \mathrm{~mm}$ ) was almost negligible with a chopping frequency higher than $40 \mathrm{~Hz}$ and an illumination power less than $1 \mathrm{~mW}$ (HeCd laser, 325 and $441 \mathrm{~nm}$ ). Note that whether the thermal effect exists or not can be judged by looking at the temporal evolution of tunnel current. ${ }^{12}$ STM topographs could be stably obtained under these conditions, although small modulation, $\sim 0.02 \mathrm{~nm}_{\mathrm{rms}}$ depending on the illumination and feedback conditions, could be observed due to the intermittent SPV effect. In order to obtain an SPV spectrum, the scanning was stopped and a conventional STS measurement was performed under the chopped illumination. Part of a typical result for an $n$-type $\mathrm{Si}(111)-7 \times 7$ sample (30 $\Omega \mathrm{cm}$ ) is shown in Fig. 1(a). The $I-V$ curve (red) oscillates widely between the two virtual $I-V$ curves (dotted black), corresponding to the dark and illuminated conditions. The SPV spectrum is acquired by plotting the lateral separation between these two virtual curves against the bias voltage under the dark condition, as shown in Figs. 1(b) and 1(c).

In this work, $I-V$ curve consisting of two thousand points was obtained in 1-2 s with a chopping frequency of $\sim 40 \mathrm{~Hz}$. The tunnel current for 30\%-80\% of each illuminated/dark period was averaged to extract the illuminated/dark current, which are shown by the blue and green circles, respectively, in Fig. 1(b). The gate time was introduced to eliminate artifacts due to the finite transient time of illumination during which the laser power changes from $0 \%$ to $100 \%$ and vice versa, from the displacement current as a result of capacitance change of the tunnel junction, and from the recovery time of the metal-insulatorsemiconductor (MIS) junction at the tunnel gap. ${ }^{13}$ Finally, the illuminated/dark $I-V$ curves for calculating SPV, which are shown by the dotted black lines in Fig. 1(a), are obtained by interpolating the measured points using the smoothing Downloaded 28 Apr 2004 to 130.158.147.14. Redistribution subje (a)
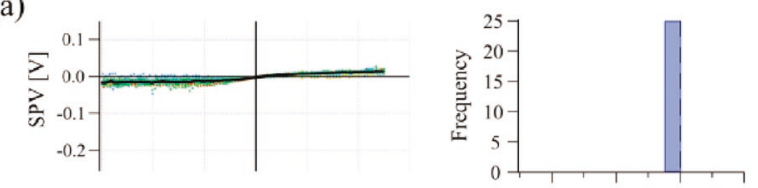

(b)
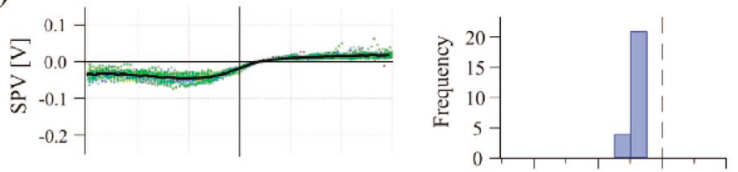

(c)
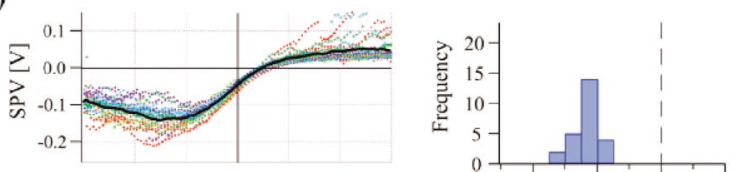

(d)
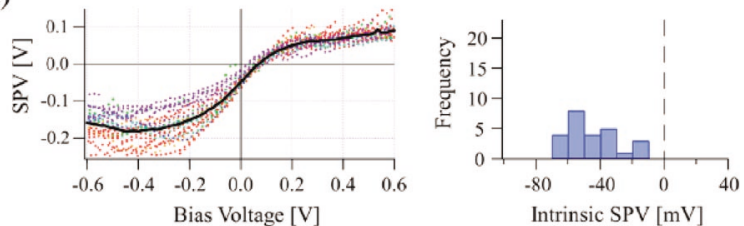

FIG. 2. (Color) SPV spectra (left column) and histograms of their $y$ sections (right column) of $p$-type $\mathrm{Si}(001)(0.03 \Omega \mathrm{cm})$. Tip-sample distance was varied by setting the bias voltage of the servo condition with a constant current setpoint of $1 \mathrm{nA}$; (a) 0.3 (b) 0.4 (c) 0.5 and (d) $0.6 \mathrm{~V}$ were used.

spline function. In our case, the precision of the $I-V$ curves was limited by a few percent of relative error due to mechanical vibration and a few picoamperes of absolute error due to broadband noise in the current signal. These errors in the tunnel current, together with the magnitude of $d I / d V$, determine the detection limit of SPV. When the tip-sample distance is longer, $d I / d V$ in the low-bias-voltage regime becomes too small. Accordingly, SPV could be measured only for the high-bias-voltage regime in such cases.

The left-hand column of Fig. 2 shows SPV spectra of a $p$-type $\mathrm{Si}(001)-2 \times 1$ surface $(0.03 \Omega \mathrm{cm})$ with various tipsample distances. The distances were determined by the servo action of conventional STM electronics under chopped illumination with changing the bias voltage from (a) 0.3 to (d) $0.6 \mathrm{~V}$ with a fixed reference current of $1.0 \mathrm{nA}$. For each tip-sample distance, 25 spectra were measured at random points on the surface. The dotted lines represent individual SPV spectra and the solid black ones represent the averaged ones. It can be seen that, even under a same servo condition, the SR-SPV spectra scatter widely. This is not because of the spatial variety of SPV but mainly because of the uncertainty in determining the tip-sample distance. The uncertainty arises from the fact that the tip-sample distance fluctuates when the servo action is active because it tries to compensate the intermitted SPV effect. Since each STS measurement is done asynchronously to the illumination, the tipsample distance differs from spectrum to another. To visualize the amount of fluctuation, the dotted spectra are colored by the tunnel current at a bias voltage of $-0.5 \mathrm{~V}$. The color scale of red to violet corresponds to $70 \%-130 \%$ of the tunnel current compared with the averaged value. In the righthand column of Fig. 2, the SR-SPV at zero bias voltage (intrinsic SR-SPV) is summarized in histograms.

In the remainder of this letter, we discuss the above re- 
sults. Figure 1 clearly shows that SR-SPV can be strongly dependent on bias voltage even for a surface with a metallic surface state, such as the $\operatorname{Si}(111)-7 \times 7$ surface. Namely, the SPV value increased linearly in the low-voltage regime $\left(\left|V_{s}\right|<0.1 \mathrm{~V}\right)$ but showed saturation in the high-voltage regimes $\left(V_{s}<-0.1 \mathrm{~V}\right.$ and $\left.0.1 \mathrm{~V}<V_{s}\right)$. The curves in these three regimes, with different slopes, were also confirmed in SR-SPV spectra for a nonmetallic $\mathrm{Si}(001)-2 \times 1$ surface, as shown in Fig. 2. These spectra cannot be simply explained in terms of the charging effect because the tunnel current changes more in the high-bias-voltage regimes than in the low-bias-voltage regime. Instead, penetration of the electric field due to the bias voltage into the subsurface region under dark condition and the amount of accumulated carriers under illuminated condition should be considered. Sommerhalter et al. reported that the surface band bending of the nonmetallic $p$-type $\mathrm{WS}_{2}$ surface under STM measurement can be qualitatively represented by a simple one-dimensional MIS junction model. ${ }^{10}$ The three regimes observed in the SPV spectra for $p$-type $\mathrm{Si}(001)$ surface can be explained in accordance with their result as follows. In the high-positivevoltage regime $\left(0.1 \mathrm{~V}<V_{s}\right)$, sufficient accumulation of majority carrier occurs under dark condition. Hence, the little band bending under the dark condition results in a small SPV. At a negative bias voltage, depletion of majority carrier occurs and the amount of surface band bending is proportional to the bias voltage under the dark condition. In the low-bias-voltage regime $\left(\left|V_{s}\right|<0.1 \mathrm{~V}\right)$, the band bending is fully relaxed by illumination, resulting in the linear dependence of SPV on bias voltage. At high negative bias voltages $\left(V_{s}<-0.1 \mathrm{~V}\right)$, the amount of band bending relaxation saturates because of the limited backdiffusion of minority carrier.

In addition to bias voltage dependence, the SPV dependence on the tip-sample distance can also be explained by the same model. In Fig. 2, a small tip-sample distance correspondings to a small SPV. In addition, the same tendency was obtained in the comparison of the dotted spectra shown in the same plot; the one with a larger current showed a smaller SPV. When the tip-sample distance is small, the photogenerated carriers tunnel efficiently into the STM tip and the accumulation of carriers at the surface region is insufficient, preventing the complete relaxation of band bending.

Furthermore, the SR-SPV at zero bias voltage was also dependent on the tip-sample distance, as shown in the righthand column of Fig. 2. This is rather surprising, since there is no tunnel current flowing between the tip and the sample in this situation. We conjecture that the very high tunnel transmission coefficient between the tip and the sample pins the surface potential, preventing the band bending relaxation. In some of the previous studies on the SPV at zero bias voltage, shortening of the tip-sample distance was attempted in order to increase the measurement accuracy. ${ }^{5,9}$ Our result revealed the fundamental limitation of such approach to measure the precise SPV at zero bias voltage.

Before summarizing the argument, we mention the high spatial resolution of LM-STS method. Figure 3 shows an SPV mapping in a color scale overlapped on a STM topograph. Silver was deposited from a tungsten filament on a clean $n$-type $\mathrm{Si}(001)$ substrate $(0.005 \Omega \mathrm{cm})$ at room tem-

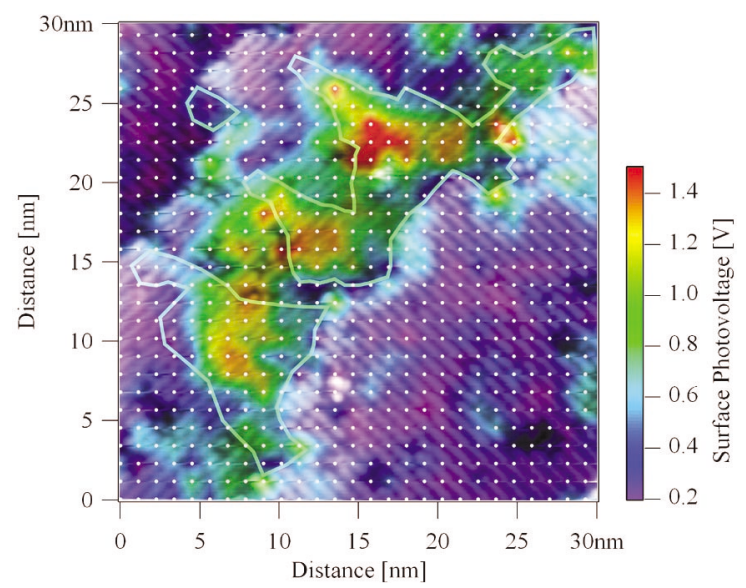

FIG. 3. (Color) SPV mapping of unsaturated $\mathrm{Ag} / \mathrm{Si}(001)-2 \times 3$ structure for $V_{s}=2.4 \mathrm{~V}$. Green lines outline the two-dimensional silver islands.

perature and annealed at $400{ }^{\circ} \mathrm{C}$. About $40 \%$ of the surface was covered by the two-dimensional silver islands of the 2 $\times 3$ structure (indicated by the green lines in Fig. 3) in accordance with literature. ${ }^{14}$ During the STM imaging over the $30 \times 30 \mathrm{~nm}$ area $\left(I_{t}=1.0 \mathrm{nA}\right.$ and $\left.V_{s}=-2.0 \mathrm{~V}\right)$ at $80 \mathrm{~K}, 27$ $\times 27$ LM-STS spectra were taken at the grid points (white dots in Fig. 3). From each spectrum, SR-SPV value for $V_{s}$ $=2.4 \mathrm{~V}$ was extracted and shown in the color scale. SPV values between the grid points were calculated by linear interpolation. Nanometer scale spatial variation of SPV due to the existence of silver islands was successfully visualized simultaneously with obtaining an atomically resolved STM topograph. The detail will be reported elsewhere. ${ }^{15}$

In summary, LM-STS was proposed as an easily accessible and very powerful method for investigating SR-SPV under various experimental conditions. Unlike previous methods, it can obtain not only the SR-SPV spectrum but also the entire illuminated/dark $I-V$ characteristics with a constant tip-sample distance. With this method, it was shown that the experimentally obtained SR-SPV is dependent on the bias voltage for a metallic $\mathrm{Si}(111)$ surface, and on the tip-sample distance for a semiconductive $\mathrm{Si}(001)$ surface even at zero bias voltage.

${ }^{1}$ S. Grafström, J. Appl. Phys. 91, 1717 (2002).

${ }^{2}$ R. J. Hamers and K. Markert, J. Vac. Sci. Technol. A 8, 3524 (1990).

${ }^{3}$ Y. Kuk, R. S. Becker, P. J. Silverman, and G. P. Kochanski, Phys. Rev. Lett. 65, 456 (1990).

${ }^{4}$ Y. Kuk, R. S. Becker, P. J. Silverman, and G. P. Kochanski, J. Vac. Sci. Technol. B 9, 545 (1991).

${ }^{5}$ T. Hagen, S. Grafström, J. Kowalski, and R. Neumann, Appl. Phys. A: Mater. Sci. Process. A66, S973 (1998).

${ }^{6}$ D. G. Cahill and R. J. Hamers, J. Vac. Sci. Technol. B 9, 564 (1991). 9, 564 (1991).

${ }^{7}$ M. McEllistrem, G. Haase, D. Chen, and R. J. Hamers, Phys. Rev. Lett. 70, 2471 (1993).

${ }^{8}$ D. Gorelik, S. Aloni, and G. Haase, Surf. Sci. 432, 265 (1999).

${ }^{9}$ D. Gorelik, S. Aloni, J. Eitle, D. Meyler, and G. Haase, J. Chem. Phys. 108, 9877 (1998).

${ }^{10}$ Ch. Sommerhalter, Th. W. Matthes, J. Boneberg, P. Leiderer, and M. Ch. Lux-Steiner, J. Vac. Sci. Technol. B 15, 1876 (1997).

${ }^{11}$ L. Kronik and Y. Shapira, Surf. Interface Anal. 31, 954 (2001).

${ }^{12}$ H. Yamamoto, I. Kamiya, and T. Takahashi, Jpn. J. Appl. Phys., Part 1 38, 3871 (1999).

${ }^{13}$ K. Maeda, M. Uota, and Y. Mera, Mater. Sci. Eng., B 42, 127 (1996).

${ }^{14}$ X. F. Lin, K. J. Wan, and J. Nogami, Phys. Rev. B 47, 10947 (1993).

${ }^{15}$ S. Yoshida, H. Oya, O. Takeuchi, and H. Shigekawa (unpublished). 